\title{
MAP MRF Joint Segmentation and Registration
}

\author{
Paul P. Wyatt* and J. Alison Noble \\ Medical Vision Laboratory, Oxford University Dept. Eng. Science \\ $\{$ wyatt, noble\}@robots.ox.ac.uk
}

\begin{abstract}
The problems of segmentation and registration are traditionally approached individually, yet the accuracy of one is of great importance in influencing the success of the other. We aim to show that more accurate and robust results may be obtained through seeking a joint solution to these linked processes. The outlined approach applies Markov random fields in the solution of a Maximum a Posteriori model of segmentation and registration. The approach is applied to synthetic and real MRI data.
\end{abstract}

\section{Introduction}

Two of the most fundamental problems in medical image analysis, and indeed within computer vision as a whole, are those of segmentation: processing raw data to obtain meaningful labels, and registration: putting information from multiple datasets into alignment. In most previous work the decision has been taken to separate these problems. This separation means the benefits inherent in the fusion of the data and communication between processes are lost.

Recently, interest has been growing in the potential of integrating segmentation and registration, driven by a clinical demand for more accurate and robust diagnostic tools. One instance is the min-max entropy approach of [2] for registration of $2 \mathcal{D}$ X-Ray portal and $3 \mathcal{D}$ CT images. An active contour approach is proposed in [12] where registration is based on contour propagation. We favour a Markov random field approach, within which we seek to obtain a maximum a posteriori estimate of the segmentation and registration.

Combined segmentation and registration should produce two principal advantages over their separate application. First, combination of registered datasets provides multiple measurements at a pixel/voxel. In a number of imaging modalities noise decorrelates with movement, which should allow more accurate classification from the combined statistics. Second, registration succeeds or fails based on the discriminatory ability of a similarity measure. This measure may be designed to use class information, so benefitting the registration. By improving the accuracy and stability of both processes, this method may benefit areas where each process fails separately. Additionally, the proof in appendix $\mathrm{A}$ shows that segmentation accuracy should increase for any distribution model, upon combination, so is not limited to special cases or functions.

\footnotetext{
* PW gratefully acknowledges the financial support of the UK EPSRC for funding this research.
} 


\section{Integrating Segmentation and Registration}

Our goal is to obtain the best possible estimate, in some predefined sense, for the segmentations of multiple data sets degraded by non-stationary noise, which are related through some geometric transformation, and to recover the geometric transformation. We cast this as a maximum a posteriori (MAP) estimation of the segmentation labels $\mathcal{S}$, transformation(s) $\mathcal{T}$ given $\mathrm{n}$ datasets $\mathbf{X}_{1}, \mathbf{X}_{2}, \ldots \mathbf{X}_{n}$ and pose the solution using Markov Random Fields (MRFs).

Using MRFs and Bayesian MAP estimation requires a model to be defined for the segmentation and registration processes, conditioned upon the data. This is important, as no matter how detailed the prior information available for a given class of problems, the data determines a specific instance of that problem. The choice of a prior model, for joint segmentation and registration, is also critical as it determines the expected relationship between images and, critically, image classes.

The Bayesian problem may be stated for two datasets $\mathrm{A}$ and $\mathrm{B}$ as:

$$
\mathcal{P}\left(\mathcal{S}, \mathcal{T} / \mathbf{X}_{A}, \mathbf{X}_{B}\right)=\frac{\mathcal{P}\left(\mathbf{X}_{A}, \mathbf{X}_{B} / \mathcal{S}, \mathcal{T}\right) \mathcal{P}(\mathcal{S}, \mathcal{T})}{\mathcal{P}\left(\mathbf{X}_{A}, \mathbf{X}_{B}\right)}
$$

If we assume dataset independence, equation 1 would simplify to:

$$
\mathcal{P}\left(\mathcal{S}, \mathcal{T} / \mathbf{X}_{A}, \mathbf{X}_{B}\right)=\frac{\mathcal{P}\left(\mathbf{X}_{A} / \mathcal{S}\right) \mathcal{P}\left(\mathbf{X}_{B} / \mathcal{S}\right) \mathcal{P}(\mathcal{S})}{\mathcal{P}\left(\mathbf{X}_{A}\right) \mathcal{P}\left(\mathbf{X}_{B}\right)}
$$

Our algorithm switches between equation 1 and 2 as their validity changes. Details of how this is done are provided later.

In the proposed method equation 1 and 2 are not implemented directly. Instead we expand $\mathcal{P}(\mathcal{S}, \mathcal{T})$ using Bayes' rule and take the logarithms of both sides. The denominator is dropped as it is constant for any data, giving:

$$
\ln \mathcal{P}\left(\mathcal{S}, \mathcal{T} / \mathbf{X}_{A}, \mathbf{X}_{B}\right) \propto \ln \mathcal{P}\left(\mathbf{X}_{A}, \mathbf{X}_{B} / \mathcal{S}, \mathcal{T}\right)+\ln \mathcal{P}(\mathcal{S} / \mathcal{T})+\ln \mathcal{P}(\mathcal{T})
$$

Throughout this paper, we refer primarily to joint estimation of segmentation and registration; it is therefore necessary to distinguish it from simultaneous estimation. We define simultaneous estimation as updating the estimation of both the classes and transforms relating any (two) datasets in a single step optimisation. Joint estimation may, or may not, use the same model of segmentation and registration, but alternates between updating the classes of any (two) datasets and updating the transforms between them in a two, or more, step optimisation.

To illustrate this, with respect to equation 3 , the joint scheme would b 1 ;

$$
\ln \mathcal{P}\left(\mathcal{S}_{n+1}, \mathcal{T}_{n} / \mathbf{X}_{A}, \mathbf{X}_{B}\right) \propto \mathcal{P}\left(\mathbf{X}_{A}, \mathbf{X}_{B} / \mathcal{S}_{n}, \mathcal{T}_{n}\right)+\ln \mathcal{P}\left(\mathcal{S}_{n} / \mathcal{T}_{n}\right)+\ln \mathcal{P}\left(\mathcal{T}_{n}\right)
$$

$\ln \mathcal{P}\left(\mathcal{S}_{n+1}, \mathcal{T}_{n+1} / \mathbf{X}_{A}, \mathbf{X}_{B}\right) \propto \ln \mathcal{P}\left(\mathbf{X}_{A}, \mathbf{X}_{B} / \mathcal{S}_{n+1}, \mathcal{T}_{n}\right)+\ln \mathcal{P}\left(\mathcal{S}_{n+1} / \mathcal{T}_{n}\right)+\ln \mathcal{P}\left(\mathcal{T}_{n}\right)$

and the simultaneous estimation;

$$
\ln \mathcal{P}\left(\mathcal{S}_{n+1}, \mathcal{T}_{n+1} / \mathbf{X}_{A}, \mathbf{X}_{B}\right) \propto \ln \mathcal{P}\left(\mathbf{X}_{A}, \mathbf{X}_{B} / \mathcal{S}_{n}, \mathcal{T}_{n}\right)+\ln \mathcal{P}\left(\mathcal{S}_{n} / \mathcal{T}_{n}\right)+\ln \mathcal{P}\left(\mathcal{T}_{n}\right)
$$

\footnotetext{
${ }^{1}$ Segmentation is performed first as the class labels are then used in registration.
} 
The difference between these schemes depends significantly upon the models for segmentation and registration and their interdependance. As they become more independent the differences recede. As the models become more reliant, so the gains in simultaneous estimation appear. However, joint estimation is significantly quicker than simultaneous estimation 2. More importantly, in this paper we principally consider rigid registration and consequently the registration criteria is evaluated over the image as a whole. This leads to small changes in segmentation having negligible effect.

\section{$3 \quad$ Registration of Images}

We use a class-based information theoretic criteria similar to Mutual Information 910] (MI). At each step of the Joint Segmentation and Registration (JS\&R) estimation a sub-optimal set of segmentation labels exist. Consider the following measure;

$$
\mathcal{I}_{\mathcal{S}_{A}, \mathcal{S}_{B}}=\sum_{k \in \mathcal{K}} \mathcal{P}_{k}\left(\mathcal{S}_{A}, \mathcal{S}_{B}\right) \ln \mathcal{P}_{k}\left(\mathcal{S}_{A}, \mathcal{S}_{B}\right)
$$

calculated from the joint class histogram of the two images, instead of the joint intensity histogram. This similarity measure is based upon image class and is conceptually similar to the MI entropy measure. This class based information measure is fast to calculate, as we have typically 2-5 class labels versus (approximately) 256 intensity levels. The smaller matrix size means fewer calculations are required for equation 6 compared to MI.

Equation [6 can be combined with the class pixel MAP probabilities if desired, to increase specificity, and is implementable for elastic registration. The MAP probabilities result from the MRF MAP ICM (Iterated Conditional Modes [3]) segmentation as calculated from the image data and prior image models.

Using the segmentation labels in the registration criteria allows estimation of the convergence of the registration. The K-means algorithm allows the estimation of the weightings of each distribution present in the $1 \mathcal{D}$ datasets. These weights appear, under Maximum Likelihood classification, as the row (column) sums of the match matrix of equation 6 and are similar under MAP. The coefficient of convergence can be evaluated as the ratio of the entropy of the class match matrix, at the $n^{\text {th }}$ iteration, to the match matrix where maximal correlation, i.e. a 1:1 correspondence, exists.

$$
\text { Coeff }_{\text {convergence }}=\frac{\sum_{k \in \mathcal{K}} \mathcal{P}_{k}\left(\mathcal{S}_{A, \text { cur }}, \mathcal{S}_{B, \text { cur }}\right) \ln \mathcal{P}_{k}\left(\mathcal{S}_{A, \text { cur }}, \mathcal{S}_{B, \text { cur }}\right)}{\sum_{k \in \mathcal{K}} \mathcal{P}_{k}\left(\mathcal{S}_{A, \text { max }} \mathcal{S}_{B, \text { max }}\right) \ln \mathcal{P}_{k}\left(\mathcal{S}_{A, \text { max }}, \mathcal{S}_{B, \text { max }}\right)}
$$

Equation 7 thereby allows switching between equations 1 and 2, at some user set threshold of convergence. This avoids obtaining a poor registration which will lead to decreased accuracy in the joint segmentation.

\footnotetext{
$\overline{2}$ This results simply from the number of classes to be considered. If there are $\mathcal{K}$ distributions and we allow the registration classes to be $\pm \delta \mathcal{T}$ in each dimension $\mathcal{D}$ then for joint estimation we consider $\mathcal{K}+(2 \mathcal{D}+1)$ classes and for simultaneous estimation $\mathcal{K}(2 \mathcal{D}+1)$, i.e. additive versus multiplicative.
} 


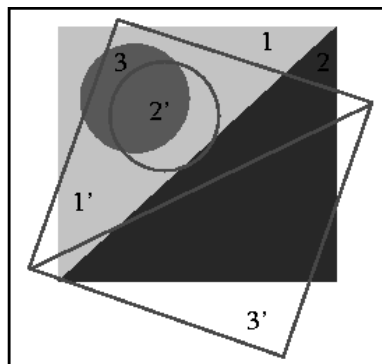

(a) During Registration

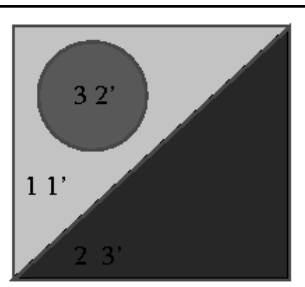

(b) Registration Completed

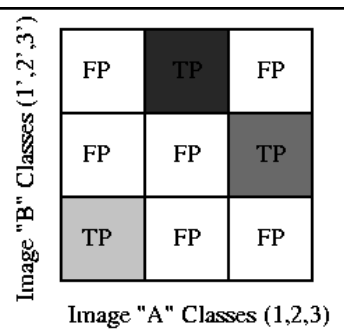

TP: True Positive FP: False Positive

(c) Class Match, During Registration

Fig. 1. Diagram of the Joint Class Histogram obtained during Registration.

\section{The Match Matrix and Segmentation}

We propose the Gaussian mixture model (GMM) to model the image data. The GMM is ideal for simply illustrating the benefit of our approach compared to sequential segmentation and registration, though other models could easily be substituted. From the individual datasets, by applying a K-Means algorithm, we obtain a $1 \mathcal{D}$ GMM for the data. As registration proceeds, classes become better correlated allowing the switch from $1 \mathcal{D}$ Gaussian intensity classes to $2 \mathcal{D}$ Gaussian intensity classes. This substantially improves the separation of the data. Figure 1 shows a diagram of the class match matrix; which forms the prior $\mathcal{P}(\mathcal{S} / \mathcal{T})$, in equation 1 As registration proceeds, the false positives tend towards a residual dependent upon classification errors. The relative weights reflect belief in different class combinations, which can be fixed if known a priori. The underlying principle is similar to Mutual Information 10, using entropy of classes rather than intensities.

The GMM required for the data model of equation 1 is formed when the registration convergence is satisfactory (equation [7). Depending whether we believe a 1:1 correspondence exists between classes in the different datasets, we can allow either a greater or smaller number of classes to be included in the GMM. This allows flexibility in modelling registration as either pure motion (1:1 correspondence) or as change between the classes in different images; for example in chemotherapy treatment of tumours.

\subsection{JS\&R Algorithms}

We assume a familiarity with Markov fields and Bayesian estimation in this section 467], particularly the Iterated Conditional Modes (ICM) algorithm 3]. Let $\hat{\mathbf{s}}$ represent class labels and $\mathbf{x}$ data. We seek, at each step;

$$
\hat{\mathbf{s}}=\arg \max _{\mathbf{s} \in \mathcal{S}}\{\mathcal{P}(\mathbf{x} \mid \mathbf{s}) \mathcal{P}(\mathbf{s})\}
$$

The JS\&R algorithm may then be summarised as follows: 
1. Starting with single datasets, perform a K-Means estimation for the model parameters. The number of classes $\mathcal{K}$ can be estimated ${ }^{3}$ or given.

2. Form a Gaussian multiresolution hierarchy. Starting at the coarsest resolution perform a MAP MRF ICM[3] segmentation using equation 2.

3. Perform Registration using the Powell method 11] and equation [6.

4. Assess registration convergence, using equation [7, Reprocess segmentation, according to equation [1, or 2] as determined by equation 7 .

5. If JS\&R converged; repeat 3-5 at next level. If done all levels finish. Else 3.

\section{Results}

Figure 2 (a,d) show two synthetic images. These have been simulated using Gaussian classes with means [25, 50, 100, 150, 200, 225] (gray levels). In (a) classes have variance $\sim 20$ and in $(\mathrm{d}) \sim 12$ (gray levels). Image $(\mathrm{d})$ is obtained from (a) through a rotation of $0.15^{c},\left(8.59^{\circ}\right)$ and translation of $[12,13]^{t}$ pixels. Figure 2 (b,e) show the segmentation of (a,d) using a GMM based MRF on each image separately. Images (c,f) show the segmentation of $(\mathrm{a}, \mathrm{d})$ using the JS\&R algorithm. The segmentation accuracie 4 are: (b) 0.908 (c) 0.956 (e) 0.977 (f) 0.986 . The transformation was correctly recovered by both registration using MI of intensities and the JS\&R algorithm to within the user specified tolerance $( \pm 0.1$ pixels translation and $\pm 0.1^{\circ}$ rotation). However, using equation [6 as opposed to MI, speeded evaluation of the registration criteria by a factor of $\sim 10$. This results from the smaller matrix size required for the class based criterion versus intensity based.

Figure 3 shows five frames, approximately $\frac{1}{2}$ a cycle, of an MRI mouse heart sequence. The first column shows the MRI images, the second the single dataset segmentations. The third and fourth columns show the paired segmentations obtained using JS\&R on pairs of adjacent images. The combined segmentation appears better than the separate; maintaining separation of left and right ventricles. The registrations obtained from conventional MI and the combined JS\&R algorithms were the same within the specified tolerance.

\section{Conclusion}

We have demonstrated that a combined approach to segmentation and registration can improve the accuracy of segmentation and speed up registration, using Markov Random Fields and Bayesian estimation. The JS\&R algorithm uses simple data and prior models suggesting an improved model should improve performance further. Although more testing is required, particularly on low quality medical data where the method should be of particular use, the benefits have been demonstrated; theoretically and practically.

\footnotetext{
${ }^{3}$ Using Minimum Description Length[8] (MDL) or otherwise.

${ }^{4}$ As the images were simulated, the actual segmentations were known. The accuracy measure used was the fraction of correctly classified pixels.
} 


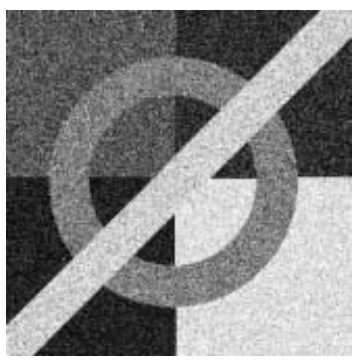

(a) Image $\mathrm{A}$

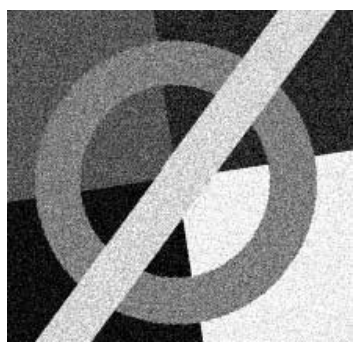

(d) Image B

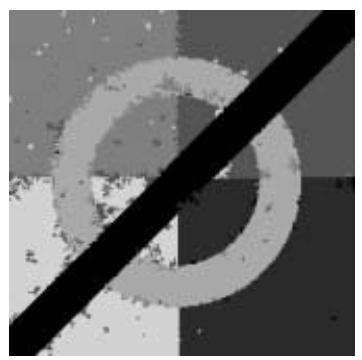

(b) Segment A:using A

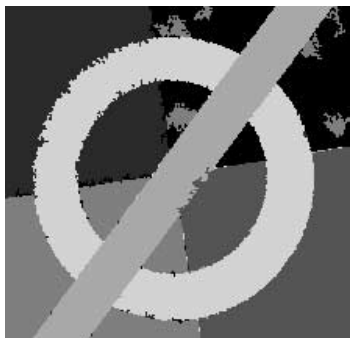

(e) Segment B:using B

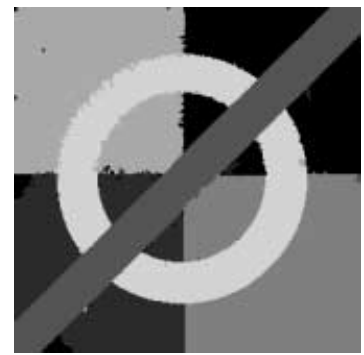

(c) Segment A:using A\&B

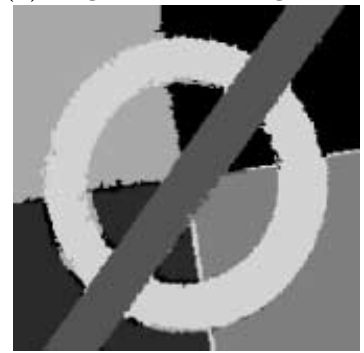

(f) Segment B:using A\&B

Fig. 2. 2 Images with 6 Classes, Gaussian Noise. (size A:250×250, B:212×221 pixels.)

\section{A Improved Separability of Functions in Higher Dimensions}

If we can prove, for any $\mathrm{N}$-dimensional function $\mathcal{F}$, that the error of Maximum Likelihood (ML) classification is upper-bounded by the $1 \mathcal{D}$ case then this proves that ML classification from the fusion of registered datasets yields equal or more accurate results than those possible for any single dataset.

Consider $1 \mathrm{D}$ functions $\mathcal{F}_{1}\left(x_{1}\right), \mathcal{F}_{2}\left(x_{1}\right)$, with ML error $\delta \mathcal{E}_{1}$ (about point $a$ );

$$
\delta \mathcal{E}_{1}=\int_{\mathrm{a}-\delta \mathrm{a}}^{\mathrm{a}+\delta \mathrm{a}} \min \left[\mathcal{F}_{1}\left(x_{1}\right), \mathcal{F}_{2}\left(x_{1}\right)\right] \mathrm{d} x_{1}
$$

In $2 \mathrm{D}$ these form, after fusion with other data, functions $\mathcal{G}_{2,1}\left(x_{1}, x_{2}\right), \mathcal{G}_{2,2}\left(x_{1}, x_{2}\right)$. The fractional error for the same data becomes;

$$
\delta \mathcal{E}_{2}=\int_{\mathrm{a}-\delta \mathrm{a}}^{\mathrm{a}+\delta \mathrm{a}} \int_{-\infty}^{\infty} \min \left[\mathcal{G}_{2,1}\left(x_{1}, x_{2}\right), \mathcal{G}_{2,2}\left(x_{1}, x_{2}\right)\right] d x_{2} d x_{1}
$$

Iff the data is independent then $\mathcal{G}_{2,(1 \cup 2)}\left(x_{1}, x_{2}\right)=\mathcal{F}_{(1 \cup 2)}\left(x_{1}\right) \mathcal{G}\left(x_{2}\right)$ and equation 10] becomes;

$$
\delta \mathcal{E}_{2}=\int_{\mathrm{a}-\delta \mathrm{a}}^{\mathrm{a}+\delta \mathrm{a}} \int_{-\infty}^{\infty} \min \left[\mathcal{G}\left(x_{2}\right) \mathcal{F}_{1}\left(x_{1}\right), \mathcal{G}\left(x_{2}\right) \mathcal{F}_{2}\left(x_{1}\right)\right] d x_{2} d x_{1}
$$


Paired Segmentation Paired Segmentation With "T-1" Image With "T+1" Image
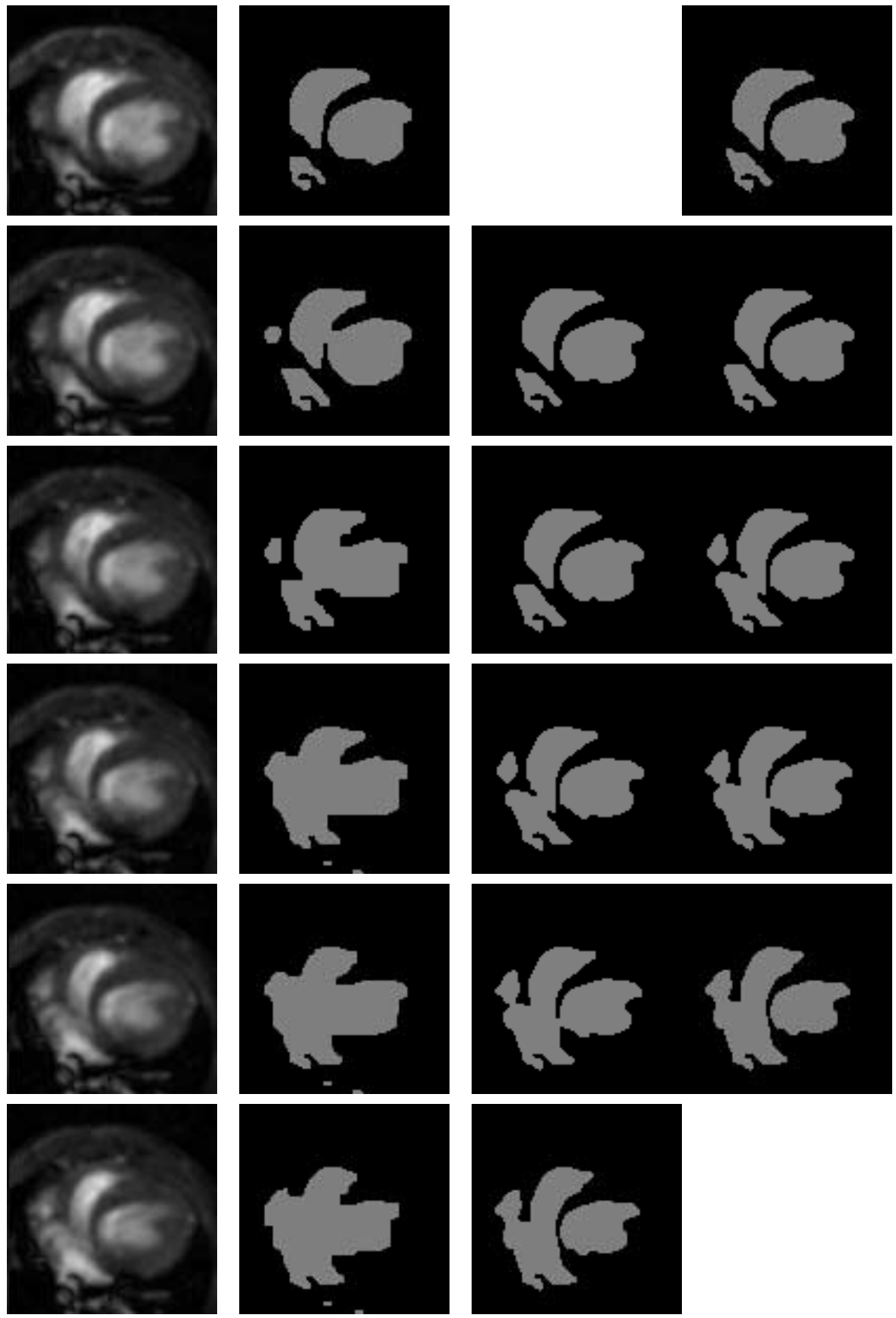

Fig. 3. Segmentations, of $\sim \frac{1}{2}$ cycle, of an MRI Mice Heart Sequence 


$$
\begin{aligned}
& =\int_{-\infty}^{\infty} \mathcal{G}\left(x_{2}\right) \mathrm{d} x_{2} \int_{\mathrm{a}-\delta \mathrm{a}}^{\mathrm{a}+\delta \mathrm{a}} \min \left[\mathcal{F}_{1}\left(x_{1}\right), \mathcal{F}_{2}\left(x_{1}\right)\right] \mathrm{d} x_{1} \\
& =\int_{\mathrm{a}-\delta \mathrm{a}}^{\mathrm{a}+\delta \mathrm{a}} \min \left[\mathcal{F}_{1}\left(x_{1}\right), \mathcal{F}_{2}\left(x_{1}\right)\right] d x_{1}
\end{aligned}
$$

Hence, the error for $2 \mathcal{D}$ is equal to that for $1 \mathcal{D}$. This also implies that the ratio $\frac{\mathcal{F}_{1}\left(x_{1}\right)}{\mathcal{F}_{2}\left(x_{1}\right)}=\mathcal{K}$, a constant. However, if $\mathcal{G}_{2,(1 \cup 2)}\left(x_{1}, x_{2}\right) \neq \mathcal{F}_{(1 \cup 2)}\left(x_{1}\right) \mathcal{G}\left(x_{2}\right)$ then the ratio $\frac{\mathcal{G}_{2,1}\left(x_{1}, x_{2}\right)}{\mathcal{G}_{2,2}\left(x_{1}, x_{2}\right)} \neq \mathcal{K}$. The maximum ML misclassification must be $\delta \mathcal{E}_{1}$, as the maximum union of $\mathcal{F}_{1}\left(x_{1}\right), \mathcal{F}_{2}\left(x_{1}\right)$ is $\min \left[\mathcal{F}_{1}\left(x_{1}\right), \mathcal{F}_{2}\left(x_{1}\right)\right]$. Consequently, extending this logic to the whole domain;

$$
\operatorname{Iff} \frac{\mathcal{G}_{\mathcal{N}, 1}\left(x_{1}, x_{2}, \cdots, \mathrm{x}_{\mathcal{N}-1}, \mathrm{x}_{\mathcal{N}}\right)}{\mathcal{G}_{\mathcal{N}, 2}\left(x_{1}, x_{2}, \cdots, \mathrm{x}_{\mathcal{N}-1}, \mathrm{x}_{\mathcal{N}}\right)} \geq \cdots \geq \frac{\mathcal{G}_{2,1}\left(x_{1}, x_{2}\right)}{\mathcal{G}_{2,2}\left(x_{1}, x_{2}\right)} \geq \frac{\mathcal{F}_{1}\left(x_{1}\right)}{\mathcal{F}_{2}\left(x_{1}\right)} \text { then } \delta \mathcal{E}_{2} \leq \delta \mathcal{E}_{1}
$$

\section{References}

1. R Bansal and LH Staib et al., A Novel Approach for the Registration of $2 D$ Portal and 3D CT Images for Treatment Setup Verification in Radiotherapy, MICCAI 1998, p1075-1086

2. R Bansal and LH Staib et al., Entropy-Based Multiple Portal to 3D CT Registration for Prostate Radiotherapy Using Iteratively Estimated Segmentation, MICCAI 1999, p567-578

3. J Besag, On the Statistical Analysis of Dirty Pictures, Journal of the Royal Statistical Society - Series B 48(3), p259-302, 1986

4. J Besag, Spatial Interaction and the Statistical Analysis of Lattice Systems, Journal of the Royal Statistical Society - Series B(36), p192-236, 1974

5. C Bishop, Neural Networks for Pattern Recognition, Oxford University Press, 1995

6. S Geman and D Geman, Stochastic Relaxation, Gibbs Distributions, and the Bayesian Restoration of Images, IEEE PAMI 1984, 6(6), p721-741.

7. DM Greig and BT Porteos and AH Seheult, Exact Maximum A Posteriori Estimation for Binary Images, Journal of the Royal Statistical Society - Series B 51(2), p271-279, 1989

8. YG Leclerc, Constructing Simple Stable Descriptions for Image Partitioning, IJCV , (3) p73-102, 1989

9. JBA Maintz and MA Viergever, A Survey of Medical Image Registration Methods, Medical Image Analysis, 2(1), p1-36,1998

10. A Roche and G Malandain and N Ayache, Unifying Maximum Likelihood Approaches in Medical Image Registration, INRIA France, Tech Report RR-3741, 1999

11. WH Press and SA Teukolsky et al, Numerical Recipes in C, Cambridge University Press, 1992

12. A Yezzi and L Zollei and T Kapur A Variational Framework for Joint Segmentation and Registration IEEE Proc. MMBIA, p44-52, 2001 